\title{
Energy-Efficient Power Allocation of Two-Hop Cooperative Systems with Imperfect Channel Estimation
}

\author{
Osama Amin ${ }^{1}$, Ebrahim Bedeer ${ }^{2}$, Mohamed H. Ahmed ${ }^{3}$, Octavia A. Dobre ${ }^{3}$ and Mohamed-Slim Alouini ${ }^{1}$ \\ ${ }^{1}$ King Abdullah University of Science and Technology (KAUST), \\ Thuwal, Makkah Province, Saudi Arabia. \\ E-mail: \{osama.amin, slim.alouini\}@kaust.edu.sa \\ ${ }^{2}$ School of Engineering, University of British Columbia, Kelowna, BC V1V 1V7, Canada. \\ E-mail: ebrahim.bedeer-mohamed@ubc.ca \\ ${ }^{3}$ Faculty of Engineering and Applied Science, Memorial University of Newfoundland, \\ St. John's, NL, A1B 3X5, Canada. \\ E-mail: \{mhahmed, odobre $\}$ mun.ca
}

\begin{abstract}
Recently, much attention has been paid to the green design of wireless communication systems using energy efficiency (EE) metrics that should capture all energy consumption sources to deliver the required data. In this paper, we formulate an accurate EE metric for cooperative two-hop systems that use the amplify-and-forward relaying scheme. Different from the existing research that assumes the availability of perfect channel state information (CSI) at the communication cooperative nodes, we assume a practical scenario, where training pilots are used to estimate the channels. The estimated CSI can be used to adapt the available resources of the proposed system in order to maximize the EE. Two estimation strategies are assumed namely disintegrated channel estimation, which assumes the availability of channel estimator at the relay, and cascaded channel estimation, where the relay is not equipped with channel estimator and only forwards the received pilot(s) in order to let the destination estimate the cooperative link. The channel estimation cost is reflected on the EE metric by including the estimation error in the signal-to-noise term and considering the energy consumption during the estimation phase. Based on the formulated EE metric, we propose an energy-aware power allocation algorithm to maximize the $\mathrm{EE}$ of the cooperative system with channel estimation. Furthermore, we study the impact of the estimation parameters on the optimized EE performance via simulation examples.
\end{abstract}

\section{INTRODUCTION}

The rapid advances in wireless communications technology increase the widespread of wireless services and applications in the daily life. To further enhance the network capacity and obtain ubiquitous service, most of the research efforts are paid to improve spectral efficiency and expand the service coverage. Recently, the energy demand has increased significantly, which lead to a dramatic increase of the information and communication technology $\mathrm{CO}_{2}$ footprint in the environment, comparable to that of the global aviation industry [1]. Furthermore, the wireless devices lifetime has to be prolonged to meet the increased communication networks data traffic requirements. These serious challenges motivate engineers to steer the attention towards the energy-aware design, which becomes a global design goal in both academic and industrial communities [2], [3]. For this purpose, the energy efficiency
(EE) metric is adopted to capture all energy consumption sources used in the communication scenario to deliver the required data successfully [4].

Cooperative communications represent a powerful technology that provides reliable transmission by increasing the throughput, expanding the coverage and mitigating the fading without the need of multiple antennas [5]. Cooperative schemes create a virtual distributed antenna system using other available nodes in the network, i.e., the relays. Therefore, single-antenna communication nodes with limited-power capability can achieve large-distance reliable communications by using cooperative schemes with a reduced cost. As a result, cooperative communications are expected to participate effectively towards energy efficient communications [6].

Recently, the EE of cooperative communications has been investigated and a general framework for the energy-efficient relaying techniques is discussed in [6]. The energy efficient resource allocation problem is investigated for the decodeand-forward (DF) in [7]-[12], and for the amplify-and-forward (AF) in [13]-[20].

Designing energy-efficient AF relaying systems based on imperfect or limited channel state information (CSI) is a challenging and interesting problem [18]-[20]. In [18], Zappone et al. explored the power allocation problem of two-hop multipleinput multiple-output AF cooperative system using statistical CSI. The same authors extended the problem to include the direct link and computed the optimal power of both source and relay in addition to the optimal conditions of beamforming that maximize the EE in [19]. In [20], Le et al. assumed a twohop AF relaying for the downlink multiuser communications, which uses one bit feedback. The authors considered a fixed data rate for multiple users, where the suggested centralized and distributed strategies notify the base station if the received signal-to-noise ratio (SNR) is below a certain threshold. To the best of our knowledge, there are no research studies for the EE of cooperative systems based on estimated CSI.

The contribution of this paper is three-fold: i) studying the EE for a relay-assisted AF system based on two channels estimation strategies, namely disintegrated channel estimation 
(DCE) and cascaded channel estimation (CCE); ii) formulating an accurate EE metric that captures the channel estimation impact in the SNR and in the total power consumption; iii) proposing an energy-aware algorithm that allocates the power of the source and the relay nodes to maximize the EE of the proposed system.

Notation: $(.)^{\mathrm{T}},(.)^{*}$, and $|$.$| denote transpose, conjugate, and$ absolute value operations, respectively. $\mathbb{E}$ denotes expectation. Bold upper-case letters denote the matrices and bold lowercase letters denote the vectors. $\mathbf{1}_{p}$ is a vector of ones with length $p$.

\section{SYSTEM MOdEL}

We assume a cooperative system consisting of a source node $(S)$, a destination node $(D)$, and a half-duplex relay node $(R)$. The source node sends its data to the destination node via the relay, where the direct link channel is assumed to be not available at the destination due to high shadowing and fading effects. We assume quasi static block fading channel, where the channel is constant over a block of data. The relay node is assumed to work in the AF mode through two orthogonal transmission phases, called broadcasting and relaying. In the first phase, $S$ broadcasts its data to $R$ and in the second phase, $S$ remains silent, while $R$ transmits an amplified version of the signal (after proper normalization) to $D$. Prior to the two phases of data transmission, a sequence of pilots is sent via the cooperative links to estimate the channels and adapt the $S$ and $R$ powers accordingly. For channel estimation purpose, we consider two channel estimation strategies: DCE and CCE that use linear minimum mean square error (LMMSE) estimator [21]. We employ these two strategies according to the availability of channel estimator at $R$. Based on the estimated CSI, the power of both $S$ and $R$ is allocated to maximize the EE, as it will be discussed in the next sections. We assume the channels of the cooperative link modelled as complex Gaussian distribution random variables.

\section{A. Disintegrated Channel Estimation (DCE)}

The DCE strategy is suitable when the relay is equipped with a channel estimator. As such, the channel estimation occurs in two phases, broadcast channel estimation phase and relying channel estimation phase. In the first phase, the source sends $N$ pilots and the relay receives them as

$$
\mathbf{r}_{\mathrm{R}}=\mathbf{x}_{\mathrm{SR}} h_{\mathrm{SR}}+\mathbf{n}_{\mathrm{R}},
$$

where $\mathbf{r}_{\mathrm{R}}$ is the received signal vector of length $N$ at the relay, $h_{\mathrm{SR}}$ is the fading channel coefficient of $S \rightarrow R$ link with zero mean and variance equal to $\sigma_{h_{\mathrm{SR}}}^{2}$, and $\mathbf{n}_{\mathrm{R}}$ is complex additive white Gaussian noise (AWGN) with zero mean and variance equal to $\sigma_{n}^{2}$, which is computed from $\sigma_{n}^{2}=N_{0} N_{\mathrm{f}} B$, where $N_{0}$ is the thermal noise power spectral density, $N_{\mathrm{f}}$ is the receiver noise figure and $B$ is the transmission bandwidth. The $\mathbf{x}_{\mathrm{SR}}$ coefficient is defined as

$$
\mathbf{x}_{\mathrm{SR}}=\sqrt{G_{\mathrm{SR}} P_{\mathrm{P}, \mathrm{S}}} \mathbf{1}_{N},
$$

where $P_{\mathrm{P}, \mathrm{S}}$ is the power of a single pilot transmitted from the source and $G_{\mathrm{SR}}$ is the large scale power coefficient that represents the path loss and is modelled as $G_{\mathrm{SR}}=$ $\left(4 \pi d_{0} / \lambda\right)^{-2}\left(d_{\mathrm{SR}} / d_{0}\right)^{-\nu}$ [22], where $d_{\mathrm{SR}}$ is the distance between $S$ and $R, d_{0}$ is the reference distance, $\lambda$ is the wavelength and $\nu$ is the path loss exponent. In the first estimation phase, the relay estimates $h_{\mathrm{SR}}$ based on the LMMSE estimation as [21]

$$
\hat{h}_{\mathrm{SR}}=\frac{\sigma_{h_{\mathrm{SR}}}^{2}}{\sigma_{n}^{2}+N G_{\mathrm{SR}} P_{\mathrm{P}, \mathrm{S}} \sigma_{h_{\mathrm{SR}}}^{2}} \mathbf{x}_{\mathrm{SR}}^{T} \mathbf{r}_{\mathrm{R}} .
$$

The mean square error (MSE) of $\hat{h}_{\mathrm{SR}}$ is expressed as [1]

$$
\sigma_{\mathrm{e}, \mathrm{SR}}^{2}=\frac{\sigma_{h_{\mathrm{SR}}}^{2} \sigma_{n}^{2}}{\sigma_{n}^{2}+N G_{\mathrm{SR}} P_{\mathrm{P}, \mathrm{S}} \sigma_{h_{\mathrm{SR}}}^{2}} .
$$

In the relaying estimation phase, the relay sends $N$ pilots to the destination to estimate the $h_{\mathrm{RD}}$ channel. The received pilot(s) signal at the destination is expressed as

$$
\mathbf{r}_{\mathrm{D}}=\mathbf{x}_{\mathrm{RD}} h_{\mathrm{RD}}+\mathbf{n}_{\mathrm{D}},
$$

where $\mathrm{x}_{\mathrm{RD}}=\sqrt{G_{\mathrm{RD}} P_{\mathrm{P}, \mathrm{R}}} 1_{N}, h_{\mathrm{RD}}$ is the fading channel for the $R \rightarrow D$ link with zero mean and variance of $\sigma_{h_{\mathrm{RD}}}^{2}$, $G_{\mathrm{RD}}$ is the corresponding path loss fading power coefficient, where $G_{\mathrm{RD}}=\left(4 \pi d_{0} / \lambda\right)^{-2}\left(d_{\mathrm{RD}} / d_{0}\right)^{-\nu}, d_{\mathrm{RD}}$ is the distance between $R$ and $D, P_{\mathrm{P}, \mathrm{R}}$ is the power of the transmitted pilot from the relay, and $\mathbf{n}_{\mathrm{D}}$ is the associated complex AWGN vector with zero mean and variance $\sigma_{n}^{2}$. The LMMSE of the $R \rightarrow D$ channel estimate is computed from [21]

$$
\hat{h}_{\mathrm{RD}}=\frac{\sigma_{h_{\mathrm{RD}}}^{2}}{\sigma_{n}^{2}+N G_{\mathrm{RD}} P_{\mathrm{P}, \mathrm{R}} \sigma_{h_{\mathrm{RD}}}^{2}} \mathbf{x}_{\mathrm{RD}}^{T} \mathbf{r}_{\mathrm{D}},
$$

and the MSE of $\hat{h}_{\mathrm{RD}}$ is expressed as [21]

$$
\sigma_{\mathrm{e}, \mathrm{RD}}^{2}=\frac{\sigma_{h_{\mathrm{RD}}}^{2} \sigma_{n}^{2}}{\sigma_{n}^{2}+N G_{\mathrm{RD}} P_{\mathrm{P}, \mathrm{R}} \sigma_{h_{\mathrm{RD}}}^{2}} .
$$

Thus, the cooperative channel link, i.e., $S \rightarrow R \rightarrow D$, is estimated to be $\hat{h}_{\mathrm{SR}} \hat{h}_{\mathrm{RD}}$ with MSE $\sigma_{\mathrm{e}, \mathrm{DCE}}^{2}$, which is found to be [21]

$$
\begin{aligned}
\sigma_{\mathrm{e}, \mathrm{DCE}}^{2} & =\sigma_{\mathrm{e}, \mathrm{SR}}^{2} \sigma_{\mathrm{e}, \mathrm{RD}}^{2} \\
& \times\left(1+\frac{N G_{\mathrm{RD}} P_{\mathrm{P}, \mathrm{R}} \sigma_{h_{\mathrm{RD}}}^{2}}{\sigma_{n}^{2}}+\frac{N G_{\mathrm{SR}} P_{\mathrm{P}, \mathrm{S}} \sigma_{h_{\mathrm{SR}}}^{2}}{\sigma_{n}^{2}}\right) .
\end{aligned}
$$

\section{B. Cascaded Channel Estimation (CCE)}

CCE strategy is adopted for the relay that is not equipped with channel estimator. This estimation strategy occurs also in two phases, broadcast and relaying phases, but the estimation of the cooperative link is done at the destination terminal. In the first transmission phase, the relay receives the pilot(s) signal and normalizes it to $\sqrt{\mathbb{E}_{h_{\mathrm{SD}}, \mathbf{n}_{\mathrm{D}}}\left\{\mathbf{r}_{\mathrm{R}}^{H} \mathbf{r}_{\mathrm{R}}\right\}}=$ $\sqrt{G_{\mathrm{SR}} P_{\mathrm{P}, \mathrm{S}} \sigma_{h_{\mathrm{SR}}}^{2}+\sigma_{n}^{2}}$ before amplifying it with power equals $P_{\mathrm{P}, \mathrm{R}}$. At $D$, the received signal is normalized by $\sqrt{1+G_{\mathrm{RD}} P_{\mathrm{P}, \mathrm{R}} \sigma_{h_{\mathrm{RD}}}^{2} /\left(G_{\mathrm{SR}} P_{\mathrm{P}, \mathrm{S}} \sigma_{h_{\mathrm{SR}}}^{2}+\sigma_{n}^{2}\right)}$ yielding

$$
\begin{aligned}
\mathbf{r}_{\mathrm{D}}= & \sqrt{\frac{G_{\mathrm{SR}} G_{\mathrm{RD}} P_{\mathrm{P}, \mathrm{S}} P_{\mathrm{P}, \mathrm{R}}}{G_{\mathrm{SR}} P_{\mathrm{P}, \mathrm{S}} \sigma_{h_{\mathrm{SR}}}^{2}+G_{\mathrm{RD}} P_{\mathrm{P}, \mathrm{R}} \sigma_{h_{\mathrm{RD}}}^{2}+\sigma_{n}^{2}}} h_{\mathrm{SR}} h_{\mathrm{RD}} \mathbf{1}_{N} \\
& +\tilde{\mathbf{n}}_{\mathrm{D}},
\end{aligned}
$$


where $\tilde{\mathbf{n}}_{\mathrm{D}}$ is the equivalent additive noise vector at $D$ after the relaying phase and is expressed as

$$
\begin{gathered}
\tilde{\mathbf{n}}_{\mathrm{D}}=\sqrt{\frac{1}{G_{\mathrm{SR}} P_{\mathrm{P}, \mathrm{S}} \sigma_{h_{\mathrm{SR}}}^{2}+G_{\mathrm{RD}} P_{\mathrm{P}, \mathrm{R}} \sigma_{h_{\mathrm{RD}}}^{2}+\sigma_{n}^{2}}} \times \\
\left(\sqrt{G_{\mathrm{RD}} P_{\mathrm{P}, \mathrm{R}}} h_{\mathrm{RD}} \mathbf{n}_{\mathrm{R}}+\sqrt{G_{\mathrm{SR}} P_{\mathrm{P}, \mathrm{S}} \sigma_{h_{\mathrm{SR}}}^{2}+\sigma_{n}^{2}} \mathbf{n}_{\mathrm{D}}\right) .
\end{gathered}
$$

The MSE of $\hat{h}_{\mathrm{SRD}}$ is computed from [21]

$$
\hat{h}_{\mathrm{SRD}}=\frac{\sigma_{h_{\mathrm{SR}}}^{2} \sigma_{h_{\mathrm{RD}}}^{2}}{\sigma_{n}^{2}+N G_{\mathrm{SRD}} P_{\mathrm{P}, \mathrm{S}} P_{\mathrm{P}, \mathrm{R}} \sigma_{h_{\mathrm{SR}}}^{2} \sigma_{h_{\mathrm{RD}}}^{2}} \mathbf{x}_{\mathrm{SRD}}^{T} \mathbf{r}_{\mathrm{D}},
$$

where $G_{\mathrm{SRD}}$ is defined as

$$
G_{\mathrm{SRD}}=\frac{G_{\mathrm{SR}} G_{\mathrm{RD}}}{G_{\mathrm{SR}} P_{\mathrm{P}, \mathrm{S}} \sigma_{h_{\mathrm{SR}}}^{2}+G_{\mathrm{RD}} P_{\mathrm{P}, \mathrm{R}} \sigma_{h_{\mathrm{RD}}}^{2}+\sigma_{n}^{2}} .
$$

The estimation MSE for the $S \rightarrow R \rightarrow D$ channel for the CCE strategy is expressed as [21]

$$
\sigma_{\mathrm{e}, \mathrm{CCE}}^{2}=\frac{\sigma_{h_{\mathrm{SR}}}^{2} \sigma_{h_{\mathrm{RD}}}^{2} \sigma_{n}^{2}}{\sigma_{n}^{2}+N G_{\mathrm{SRD}} P_{\mathrm{P}, \mathrm{S}} P_{\mathrm{P}, \mathrm{R}} \sigma_{h_{\mathrm{SR}}}^{2} \sigma_{h_{\mathrm{RD}}}^{2}} .
$$

\section{ENERGy EFFICIENCY OF Two-Hop AF COOPERATIVE SYSTEM WITH ESTIMATED CSI}

In this section, we formulate the EE metric for the twohop AF cooperative system by taking into account the channel estimation cost. This cost appears as a reduction in the SNR that results from the estimation error and an increase in the energy consumption as a result of pilot(s) transmission and reception. The EE metric is defined as

$$
\eta_{\mathrm{EE}}=\frac{\eta_{\mathrm{SE}}}{P_{\text {Total }}} \quad \mathrm{bit} / \mathrm{Hz} / \text { joule, }
$$

where $\eta_{\mathrm{SE}}$ is the spectral efficiency of the transmitted data and $P_{\text {Total }}$ is the total power consumption of all participated nodes during the packet transmission, which includes the consumed power for data and pilots during transmission and reception. The spectral efficiency of the two-hop AF cooperative system is expressed in terms of the source and relay powers, $P_{\mathrm{S}}$ and $P_{\mathrm{R}}$, respectively, as

$$
\eta_{\mathrm{SE}}=\frac{1}{2} \log _{2}\left(1+\frac{\gamma_{\mathrm{SRD}} P_{\mathrm{S}} P_{\mathrm{R}}}{\varepsilon_{\mathrm{SRD}} P_{\mathrm{S}} P_{\mathrm{R}}+\alpha_{\mathrm{SR}} P_{\mathrm{S}}+\alpha_{\mathrm{RD}} P_{\mathrm{R}}+1}\right),
$$

where $\gamma_{\mathrm{SRD}}$ is the channel-to-noise ratio (CNR) of the cooperative link, i.e., $S \rightarrow R \rightarrow D$ link, and is written as

$$
\gamma_{\mathrm{SRD}}=\left\{\begin{array}{cc}
\frac{G_{\mathrm{SR} G} G_{\mathrm{RD}}\left|\hat{h}_{\mathrm{SR}}\right|^{2}\left|\hat{h}_{\mathrm{RD}}\right|^{2}}{\sigma_{n}^{4}} & \text { for DCE } \\
\frac{G_{\mathrm{SR}} G_{\mathrm{R}}\left|\hat{h}_{\mathrm{SRD}}\right|^{2}}{\sigma_{n}^{4}} & \text { for CCE }
\end{array},\right.
$$

$\alpha_{\mathrm{SR}}$ is the CNR of the $S \rightarrow R$ link and is expressed as

$$
\alpha_{\mathrm{SR}}=\left\{\begin{array}{ll}
\frac{G_{\mathrm{SR}}\left|\hat{h}_{\mathrm{SR}}\right|^{2}}{\sigma_{2}^{2}} & \text { for DCE } \\
\frac{G_{\mathrm{SR}} \sigma_{h_{\mathrm{SR}}}}{\sigma_{n}^{2}} & \text { for CCE }
\end{array},\right.
$$

$\alpha_{\mathrm{RD}}$ is the CNR of the $R \rightarrow D$ link and is expressed as

$$
\alpha_{\mathrm{RD}}=\left\{\begin{array}{ll}
\frac{G_{\mathrm{RD}}\left|\hat{h}_{\mathrm{RD}}\right|^{2}}{\sigma_{n}^{2}} & \text { for DCE } \\
\frac{G_{\mathrm{RD}} \sigma_{\mathrm{RD}}^{2}}{\sigma_{n}^{2}} & \text { for CCE }
\end{array},\right.
$$

and $\varepsilon_{\mathrm{SRD}}$ is the estimation MSE-to-noise ratio and is expressed as

$$
\varepsilon_{\mathrm{SRD}}=\left\{\begin{array}{ll}
\frac{G_{\mathrm{SR}} G_{\mathrm{RD}} \sigma_{\mathrm{e}, \mathrm{DCE}}^{2}}{\sigma_{n}^{4}} & \text { for DCE } \\
\frac{G_{\mathrm{SR}} G_{\mathrm{R} D} \sigma_{e, \mathrm{CCE}}^{2}}{\sigma_{n}^{4}} & \text { for CCE }
\end{array} .\right.
$$

We observe that the unavailability of individual link channel estimates in CCE forces us to use the expected values of the channel gain instead of the actual value [21], as can be seen in (17) and (18).

The spectral efficiency can be approximated as

$$
\eta_{\mathrm{SE}} \approx \frac{1}{2} \log _{2}\left(1+\gamma_{\mathrm{SRD}} f\left(P_{\mathrm{S}}, P_{\mathrm{R}}\right)\right),
$$

where $f\left(P_{\mathrm{S}}, P_{\mathrm{R}}\right)=P_{\mathrm{S}} P_{\mathrm{R}} /\left(\varepsilon_{\mathrm{SRD}} P_{\mathrm{S}} P_{\mathrm{R}}+\alpha_{\mathrm{SR}} P_{\mathrm{S}}+\alpha_{\mathrm{RD}} P_{\mathrm{R}}\right)$.

The total power consumed during the proposed two-hop AF communication scenario is given by

$$
P_{\text {Total }}=\frac{1}{2}\left(\kappa_{\mathrm{S}} P_{\mathrm{S}}+\kappa_{\mathrm{R}} P_{\mathrm{R}}\right)+P_{\mathrm{c}},
$$

where $\kappa_{\mathrm{S}}$ and $\kappa_{\mathrm{R}}$ are constants that depend on the power amplifier efficiency of $S$ and $R$, respectively, and $P_{\mathrm{c}}$ is the power consumed in all circuits, which can be written as

$$
P_{\mathrm{c}}=\frac{1}{2}\left(P_{\mathrm{ct}, \mathrm{S}}+P_{\mathrm{cr}, \mathrm{R}}+P_{\mathrm{ct}, \mathrm{R}}+P_{\mathrm{cr}, \mathrm{D}}\right)+P_{\mathrm{c}, \mathrm{CE}} .
$$

The subscripts ct and cr denote the transmission and reception circuits, respectively, and $P_{\mathrm{c}, \mathrm{CE}}$ is the power consumed during the channel estimation phase, which can be expressed as

$$
\begin{aligned}
P_{\mathrm{c}, \mathrm{CE}}=\frac{\rho}{2}\left(\kappa_{\mathrm{S}} P_{\mathrm{P}, \mathrm{S}}\right. & \left.+P_{\mathrm{ct}, \mathrm{S}}+P_{\mathrm{cr}, \mathrm{R}}\right) \\
& +\frac{\rho}{2}\left(\kappa_{\mathrm{R}} P_{\mathrm{P}, \mathrm{R}}+P_{\mathrm{ct}, \mathrm{R}}+P_{\mathrm{cr}, \mathrm{D}}\right),
\end{aligned}
$$

where $\rho$ represents the pilots-to-data ratio in the transmitted packet.

We observe from the EE expression in (14) and related equations that improving the channel estimation via either increasing the pilots' power or the number of pilots can improve significantly the SNR, but it does not necessarily improve the EE. In addition, we need to adjust the data power allocated to $S$ and $R$ to maximize the $\mathrm{EE}$ for a given estimation quality. In the following section, we focus on this problem and propose an algorithm to solve the EE maximization problem for the two-hop AF cooperative system working with either CCE or DCE.

\section{Energy-Efficient Power Allocation}

In this section, we assume fixed known channel estimation parameters represented by the number of pilots, pilot(s) power and pilot(s) density with respect to the data, and we optimize the data power at both $S$ and $R$ to maximize the EE. The power allocation problem is defined based on (20) as

$$
\begin{array}{cl}
\max _{P_{\mathrm{S}}, P_{\mathrm{R}}} & \eta_{\mathrm{EE}} \\
\text { subject to } & 0<P_{\mathrm{S}} \leq P_{\mathrm{S}, \max } \\
& 0<P_{\mathrm{R}} \leq P_{\mathrm{R}, \max },
\end{array}
$$

where $P_{\mathrm{S}, \max }$ and $P_{\mathrm{R}, \max }$ are the maximum power budget of $S$ and $R$, respectively. To solve this nonlinear fractional problem, we use the Dinkelbach method [23] to convert the 
non-concave fractional function into a concave function, which leads to the following equivalent optimization problem

$$
\begin{array}{cl}
\max _{P_{\mathrm{S}}, P_{\mathrm{R}}} & \mathcal{F}_{\mathrm{EE}}\left(P_{\mathrm{S}}, P_{\mathrm{R}}\right) \\
\text { subject to } & 0<P_{\mathrm{S}} \leq P_{\mathrm{S}, \max } \\
& 0<P_{\mathrm{R}} \leq P_{\mathrm{R}, \max },
\end{array}
$$

where $\mathcal{F}_{\mathrm{EE}}\left(P_{\mathrm{S}}, P_{\mathrm{R}}\right)$ is defined as

$$
\begin{aligned}
\mathcal{F}_{\mathrm{EE}} & \left(P_{\mathrm{S}}, P_{\mathrm{R}}\right)=\left(\frac{1}{\log 2}\right) \log \left(1+\gamma_{\mathrm{SRD}} f\left(P_{\mathrm{S}}, P_{\mathrm{R}}\right)\right) \\
- & q\left(\kappa_{\mathrm{S}} P_{\mathrm{S}}+\kappa_{\mathrm{R}} P_{\mathrm{R}}+P_{\mathrm{c}}\right),
\end{aligned}
$$

and $q$ is a constant that is equal to the maximum EE value, i.e., $q=\max \eta_{\mathrm{EE}}$. In other words, if $\left(P_{\mathrm{S}}^{*}, P_{\mathrm{R}}^{*}\right)=\arg \max \eta_{\mathrm{EE}}$, then $\mathcal{F}_{\mathrm{EE}}\left(P_{\mathrm{S}}^{*}, P_{\mathrm{R}}{ }^{*}\right)=0$ for a unique value of $q$ [23].

To study the concavity of $\mathcal{F}_{\mathrm{EE}}\left(P_{\mathrm{S}}, P_{\mathrm{R}}\right)$, we first study the concavity of $f\left(P_{\mathrm{S}}, P_{\mathrm{R}}\right)$ by computing its Hessian matrix, which is found to be written as

$$
\begin{aligned}
& \nabla^{2} f\left(P_{\mathrm{S}}, P_{\mathrm{R}}\right)=\frac{-2}{\left(\varepsilon_{\mathrm{SRD}} P_{\mathrm{S}} y+\alpha_{\mathrm{SR}} P_{\mathrm{S}}+\alpha_{\mathrm{RD}} P_{\mathrm{R}}\right)^{3}} \times \\
& {\left[\begin{array}{cc}
\alpha_{\mathrm{RD}} P_{\mathrm{R}}^{2}\left(\varepsilon_{\mathrm{SRD}} P_{\mathrm{R}}+\alpha_{\mathrm{SR}}\right) & -\alpha_{\mathrm{SR}} \alpha_{\mathrm{RD}} P_{\mathrm{R}} P_{\mathrm{S}} \\
-\alpha_{\mathrm{SR}} \alpha_{\mathrm{RD}} P_{\mathrm{R}} P_{\mathrm{S}} & \alpha_{\mathrm{SR}} P_{\mathrm{S}}^{2}\left(\varepsilon_{\mathrm{SRD}} P_{\mathrm{S}}+\alpha_{\mathrm{RD}}\right)
\end{array}\right] .}
\end{aligned}
$$

Then we find that for any real vector $\mathbf{z}=\left[\begin{array}{ll}z_{1} & z_{2}\end{array}\right]$

$$
\begin{gathered}
\mathbf{z}^{T} \nabla^{2} f\left(P_{\mathrm{S}}, P_{\mathrm{R}}\right) \mathbf{z}=-\frac{2 \alpha_{\mathrm{SR}} \alpha_{\mathrm{RD}}}{\left(\varepsilon_{\mathrm{SRD}} P_{\mathrm{S}}+\alpha_{\mathrm{SR}} P_{\mathrm{S}}+\alpha_{\mathrm{RD}} P_{\mathrm{R}}\right)^{3}} \times \\
{\left[\left(P_{\mathrm{R}} z_{1}-P_{\mathrm{S}} z_{2}\right)^{2}+\frac{\varepsilon_{\mathrm{SRD}}}{\alpha_{\mathrm{SR}}} P_{\mathrm{R}}^{2} z_{1}^{2}+\frac{\varepsilon_{\mathrm{SRD}}}{\alpha_{\mathrm{RD}}} P_{\mathrm{S}}^{3} z_{2}^{2}\right]<0,}
\end{gathered}
$$

which proves the concavity of $f\left(P_{\mathrm{S}}, P_{\mathrm{R}}\right)$. Thus, based on the operations that preserve concavity [24], $\mathcal{F}_{\mathrm{EE}}\left(P_{\mathrm{S}}, P_{\mathrm{R}}\right)$ can be proven to be concave.

To solve (25), we differentiate $\mathcal{F}_{\mathrm{EE}}$ with respect to $P_{\mathrm{S}}$ and equate the result to zero obtaining

$$
\frac{\alpha_{\mathrm{RD}} \gamma_{\mathrm{SRD}} f^{2}\left(P_{\mathrm{S}}, P_{\mathrm{R}}\right)}{P_{\mathrm{S}}^{2}\left(1+\gamma_{\mathrm{SRD}} f\left(P_{\mathrm{S}}, P_{\mathrm{R}}\right)\right)}-q \kappa_{\mathrm{S}} \log 2=0 .
$$

Similarly, by differentiating $\mathcal{F}_{\mathrm{EE}}$ with respect to $P_{\mathrm{R}}$ and equating the result to zero, we obtain

$$
\frac{\gamma_{\mathrm{SRD}} \alpha_{\mathrm{SR}} f^{2}\left(P_{\mathrm{S}}, P_{\mathrm{R}}\right)}{P_{\mathrm{R}}^{2}\left(1+\gamma_{\mathrm{SRD}} f\left(P_{\mathrm{S}}, P_{\mathrm{R}}\right)\right)}-q \kappa_{\mathrm{R}} \log 2=0 .
$$

Based on (29) and (30), $P_{\mathrm{R}}$ can be expressed in terms of $P_{\mathrm{S}}$ as

$$
P_{\mathrm{R}}=\beta P_{\mathrm{S}}, \quad \beta=\sqrt{\frac{\alpha_{\mathrm{SR}} \kappa_{\mathrm{S}}}{\alpha_{\mathrm{RD}} \kappa_{\mathrm{R}}}} .
$$

The optimization problem in (25) can be simplified and formulated to an equivalent problem in terms of $P_{\mathrm{S}}$ as follows

$$
\begin{aligned}
& \max _{P_{\mathrm{S}}} \mathcal{F}\left(P_{\mathrm{S}}\right) \\
& \text { subject to } 0<P_{\mathrm{S}} \leq P_{\max },
\end{aligned}
$$

where $P_{\max }=\min \left(P_{\mathrm{S}, \max }, \frac{1}{\beta} \mathrm{R}, \max \right)$ and the modified objective function $\mathcal{F}\left(P_{\mathrm{S}}\right)$ is expressed as

$$
\begin{aligned}
\mathcal{F}\left(P_{\mathrm{S}}\right)= & \frac{1}{\log 2} \log \left(1+\frac{\gamma_{\mathrm{SRD}} \beta P_{\mathrm{S}}}{\varepsilon_{\mathrm{SRD}} \beta P_{\mathrm{S}}+\alpha_{\mathrm{SR}}+\alpha_{\mathrm{RD}} \beta}\right) \\
& -q\left(\left(\kappa_{\mathrm{S}}+\kappa_{\mathrm{R}} \beta\right) P_{\mathrm{S}}+P_{\mathrm{c}}\right),
\end{aligned}
$$

TABLE I

SIMULATION PARAMETERS.

\begin{tabular}{|c|c|c|}
\hline$P_{\mathrm{ct}, \mathrm{S}}=P_{\mathrm{ct}, \mathrm{R}}=112 \mathrm{~mW}$ & $\kappa_{\mathrm{S}}=\kappa_{\mathrm{R}}=2.88$ & $\nu=3.5$ \\
\hline$P_{\mathrm{S}, \max }=120 \mathrm{~mW}$ & $P_{\mathrm{R}, \max }=120 \mathrm{~mW}$ & $\lambda=0.12 \mathrm{~m}$ \\
\hline$P_{\mathrm{cr}, \mathrm{R}}=P_{\mathrm{cr}, \mathrm{D}}=98 \mathrm{~mW}$ & $d_{\mathrm{SD}}=300 \mathrm{~m}$ & $B=10 \mathrm{KHz}$ \\
\hline$d_{\mathrm{RD}}=0.5 d_{\mathrm{SD}} \mathrm{m}$ & $d_{\mathrm{SR}}=0.5 d_{\mathrm{SD}} \mathrm{m}$ & $d_{0}=1 \mathrm{~m}$ \\
\hline$\sigma_{h_{\mathrm{RD}}}^{2}=\sigma_{h_{\mathrm{SR}}}^{2}=1$ & $N_{\mathrm{f}}=7 \mathrm{~dB}$ & $N=1$ \\
\hline$P_{\mathrm{P}, \mathrm{S}}=P_{\mathrm{P}, \mathrm{R}}=120 \mathrm{~mW}$ & $N_{0}=-171 \mathrm{dBm} / \mathrm{Hz}$ & $\rho=N /(20-N)$ \\
\hline
\end{tabular}

where for $\left(P_{\mathrm{S}}^{*}, P_{\mathrm{R}}^{*}\right)=\arg \max \eta_{\mathrm{EE}}$, we have $\mathcal{F}\left(P_{\mathrm{S}}^{*}\right)=0$ for a unique $q$ value [23].

The equivalent simplified optimization problem is still concave problem where one can find easily that $\frac{\partial^{2} \mathcal{F}\left(P_{\mathrm{S}}\right)}{\partial P_{\mathrm{S}}^{2}} \leq 0$.

After differentiating $\mathcal{F}\left(P_{\mathrm{S}}\right)$ with respect to $P_{\mathrm{S}}$ and equating the result to zero, $P_{\mathrm{S}}$ can be computed assuming known $q$ from

$$
\begin{aligned}
P_{\mathrm{S}} & =\frac{\left(\alpha_{\mathrm{SR}}+\alpha_{\mathrm{RD}} \beta\right)}{2\left(2 \varepsilon_{\mathrm{SRD}}+\gamma_{\mathrm{SRD}}\right) \delta \beta} \times \\
& \left(\sqrt{1-4 \delta\left(1-\frac{\gamma_{\mathrm{SRD}} \beta /\left(\alpha_{\mathrm{SR}}+\alpha_{\mathrm{RD}} \beta\right)}{q\left(\kappa_{\mathrm{S}}+\kappa_{\mathrm{R}} \beta\right)(\log 2)}\right)}-1\right),
\end{aligned}
$$

where $\delta$ is defined as

$$
\delta=\frac{\left(\varepsilon_{\mathrm{SRD}}+\gamma_{\mathrm{SRD}}\right) \varepsilon_{\mathrm{SRD}}}{\left(2 \varepsilon_{\mathrm{SRD}}+\gamma_{\mathrm{SRD}}\right)^{2}} .
$$

To find the optimal solution of (24), we develop Algorithm I that uses the Dinklebach method and calculate iteratively the energy-efficient power allocation solution.

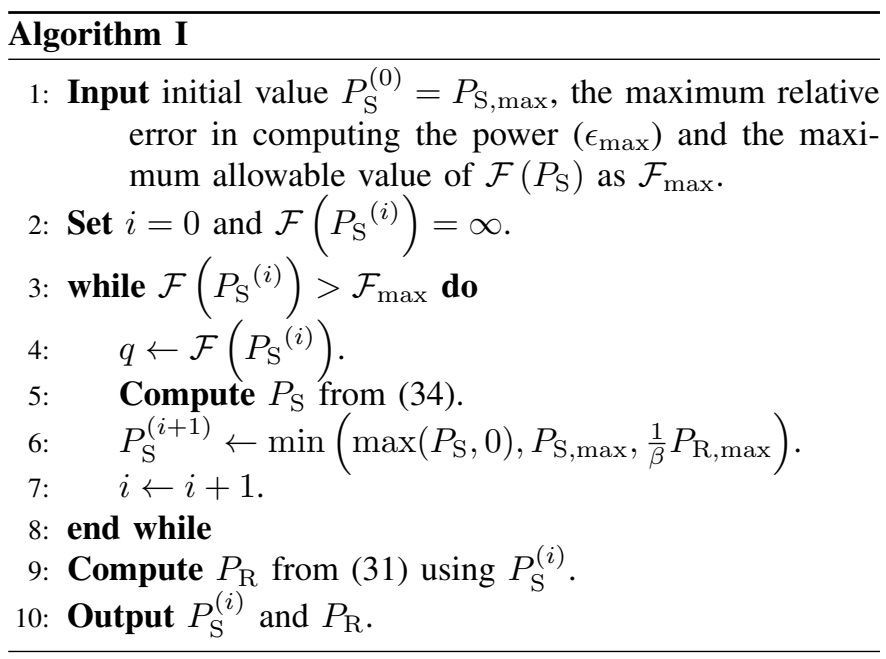

\section{Simulation Results}

In this section, we provide a numerical evaluation of the proposed energy-efficient power allocation algorithm for the two-hop AF cooperative system using the two estimation strategies, i.e., CCE and DCE. The circuits power consumption parameters are considered from [9] and listed with other simulation parameters in Table I. The simulation examples use these parameters, unless otherwise specified.

Simulation Example 1: In the first simulation example, we compare the EE of the proposed cooperative system using the exact spectral efficiency expression in (15) and the approximate expression in (20). For this purpose, we plot the EE of 


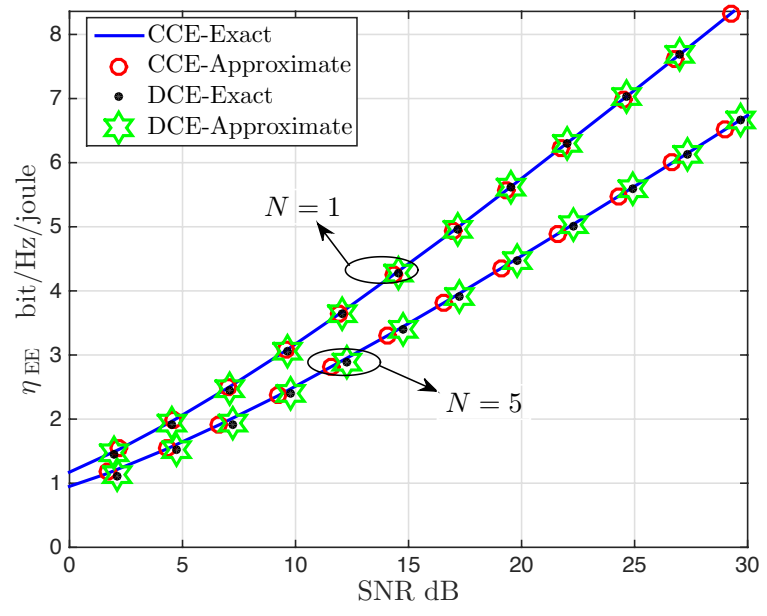

Fig. 1. Exact and approximate EE expressions versus SNR for both CCE and DCE strategies assuming different number of pilots.

both expressions versus the average end-to-end SNR in Fig. 1 for both strategies CCE and DCE assuming different number of pilots. The power terms of $S$ and $R$ are assumed to be $P_{\mathrm{S}}=P_{\mathrm{S}, \max }, P_{\mathrm{R}}=P_{\mathrm{R}, \max }$ and equal maximum power for the pilots. We observe that the approximate EE expression gives a very good match with the exact expression for both methods in different SNR regions ${ }^{1}$.

Simulation Example 2: The proposed framework considers the estimation cost into the account in designing the energy-efficient cooperative system. This example examines the importance of this consideration by comparing the energyefficient design based on perfect CSI and estimated CSI. The perfect-CSI-based design is done by assuming zero estimation error, i.e. $\varepsilon_{\mathrm{SRD}}=0$, and by neglecting the power consumed during the estimation phase, i.e., $P_{\mathrm{c}, \mathrm{CE}}=0$. To investigate the impact of channel estimation on the design, we consider three scenario with different number of pilots, $N=1,3,5$ with maximum power budget of $1.2 \mathrm{~W}$. According to the comparison results in Fig. 2, the importance of the estimatedbased increases with the increase of number of pilots.

Simulation Example 3: In this example, we study the effect of the number of pilots $(N)$ and their power on the EE performance of the two-hop AF cooperative systems. In the following simulations we assume symmetrical equal pilots power at both $S$ and $R$ and that the pilots power changes from $1 \mathrm{~mW}$ to its maximum budget value of $120 \mathrm{~mW}$. Increasing the number of pilots and/or their power is expected to improve the estimation quality for both techniques [21].In the following, we investigate whether the same performance trend is followed with EE or not. Fig. 3 shows the EE of both the CCE and DCE strategies versus the pilots power for different number of pilots. For the single pilot scenario, i.e., $N=1$, we observe that the EE performance improves with the increase of pilots' power, while this trend changes when the number of pilots increases to 3 and 5. Specifically, maximum power-optimized

\footnotetext{
${ }^{1}$ Similar results are observed also for different relay locations but are not included due to space limitations.
}

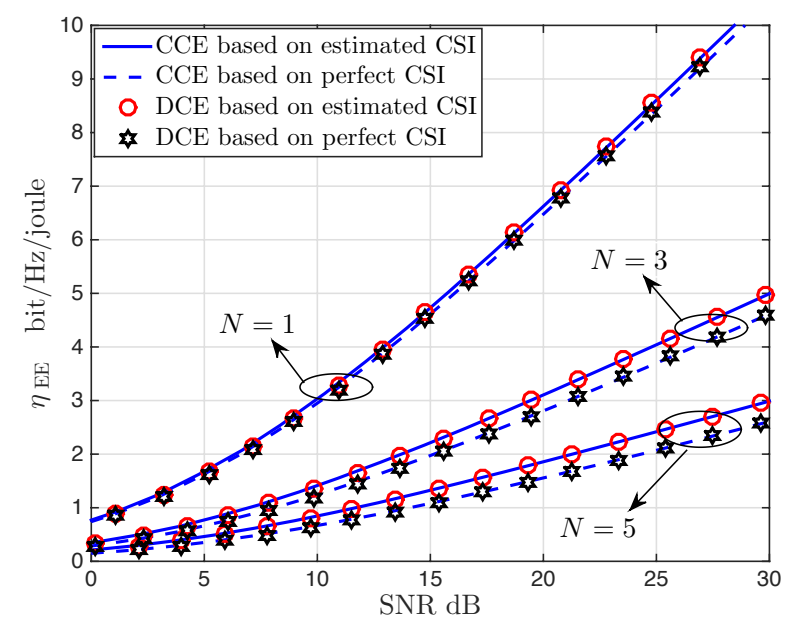

Fig. 2. Comparison between the EE design based on perfect and estimated CSI for both CCE and DCE strategies assuming different number of pilots.

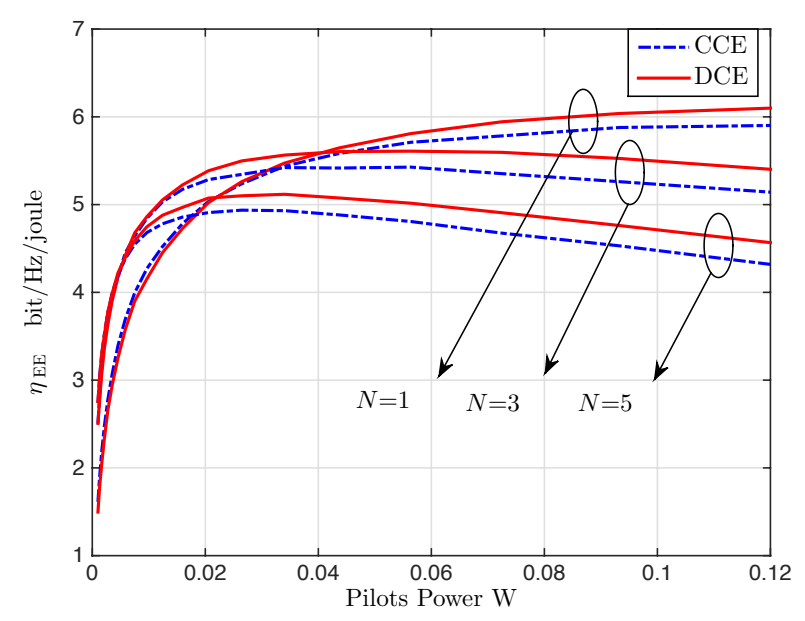

Fig. 3. EE versus the power of pilots assuming different number of pilots for both CCE and DCE strategies.

EE can be achieved at $P_{\mathrm{P}, \mathrm{S}}=P_{\mathrm{P}, \mathrm{R}}=60 \mathrm{~mW}$ for $N=3$, and $P_{\mathrm{P}, \mathrm{S}}=P_{\mathrm{P}, \mathrm{R}}=35 \mathrm{~mW}$ for $N=5$. These results indicate that there is also an optimal power value for the single pilot case, but it is expected to be more than the available budget. The existence of the optimal pilots power for maximum EE means that this power value gives a good channel estimate and the MSE can be considered acceptable in this communication scenario. The acceptable MSE for both $N=3$ and $N=5$ is approximately the same and equals $6 \times 10^{-3}$.

Simulation Example 4: Through this example, we aim to study the effect of the relay location on the EE performance. Fig. 4 plots the EE versus the relative relay location with respect to the destination for three cases that correspond to different maximum power budgets at both $S$ and $R$. Case 1 represents the assumption of symmetrical coefficients, i.e., equal systems parameters at both $S$ and $R$ as listed in Table I with $P_{\mathrm{S}, \max }=P_{\mathrm{R}, \max }=120 \mathrm{~mW}$, while Case 2 is for reduced $S$ power budget with $P_{\mathrm{S}, \max }=12 \mathrm{~mW}$, and Case 3 


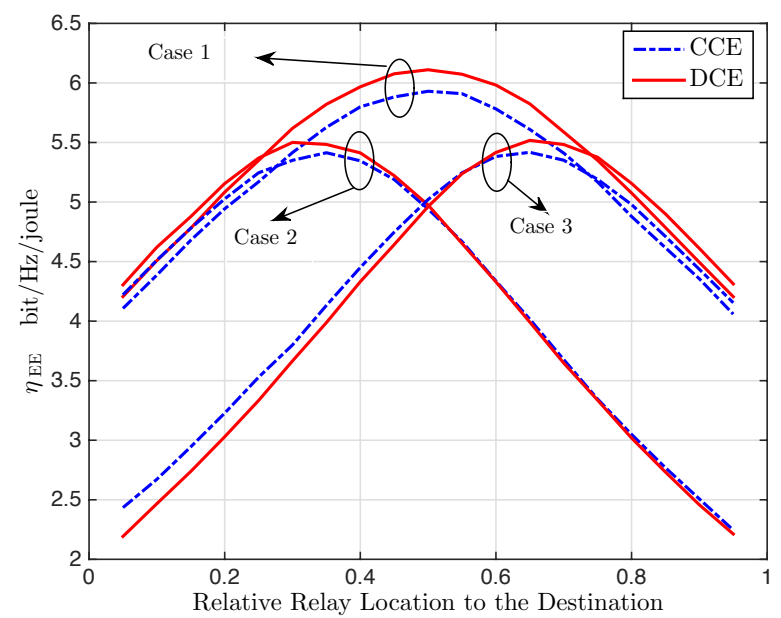

Fig. 4. EE versus the relative relay location with respect to the $S-D$ distance for both the CCE and DCE strategies.

is for reduced $R$ power budget with $P_{\mathrm{R}, \max }=12 \mathrm{~mW}$. We observe that in Case 1, the system parameters listed in Table I are symmetric; thus, the EE performance is symmetric versus relay location with maximum performance at the middle location between $S$ and $R$. As the system parameters become asymmetrical, the EE performance becomes also asymmetrical. The optimal $R$ location is near $S$ when the $S$ power budget is less than the $R$, while it it near $D$ when the $R$ power budget is less than for $S$.

\section{CONCLUSION}

In this paper, the channel estimation cost of the two-hop $\mathrm{AF}$ cooperative system is reflected in the EE metric in both reducing the SNR and increasing the power consumption. Two estimation strategies are adopted, namely CCE and DCE, depending on the availability of the channel estimator at the relay node. Then, the power allocation problem for the twohop AF cooperative systems is considered to maximize the EE assuming estimated. An energy-aware power allocation algorithm is then proposed to maximize the EE metric of the cooperative system with both estimation strategies. The EE cooperative system achieves notable energy saving at both medium and high SNR regions. Considering the channel estimation impact in the design is important when the number and/or power of pilots increase. Increasing the accuracy of the channel estimation may not always improve the EE performance; instead, it is recommended to tune the estimator to achieve an acceptable MSE from the EE perspective.

\section{REFERENCES}

[1] A. Fehske, G. Fettweis, J. Malmodin, and G. Biczok, "The global footprint of mobile communications: The ecological and economic perspective," IEEE Commun. Mag., vol. 49, no. 8, pp. 55-62, Aug. 2011.

[2] L. M. Correia, D. Zeller, O. Blume, D. Ferling, Y. Jading, I. Gódor, G. Auer, and L. Van Der Perre, "Challenges and enabling technologies for energy aware mobile radio networks," IEEE Commun. Mag., vol. 48, no. 11, pp. 66-72, Nov. 2010.
[3] C. Han, T. Harrold, S. Armour, I. Krikidis, S. Videv, P. M. Grant, H. Haas, J. S. Thompson, I. Ku, C.-X. Wang, T. A. Le, M. R. Nakhai, J. Zhang, and L. Hanzo, "Green radio: Radio techniques to enable energy-efficient wireless networks," IEEE Commun. Mag., vol. 49, no. 6, pp. 46-54, May 2011.

[4] D. Feng, C. Jiang, G. Lim, L. J. Cimini Jr, G. Feng, and G. Y. Li, "A survey of energy-efficient wireless communications," IEEE Commun. Surveys Tutorials, vol. 15, no. 1, pp. 167-178, First Quarter 2013.

[5] A. Nosratinia, T. E. Hunter, and A. Hedayat, "Cooperative communication in wireless networks," IEEE Commun. Mag., vol. 42, no. 10, pp. 74-80, Oct. 2004.

[6] O. Amin, S. Bavarian, and L. Lampe, "Cooperative techniques for energy-efficient wireless communications," in Green Radio Communication Networks. Cambridge University Press, 2012, pp. 125-149.

[7] G. G. de Oliveira Brante, M. T. Kakitani, and R. D. Souza, "Energy efficiency analysis of some cooperative and non-cooperative transmission schemes in wireless sensor networks," IEEE Trans. Commun., vol. 59, no. 10, pp. 2671-2677, Oct. 2011.

[8] N. Abuzainab and A. Ephremides, "Energy efficiency of cooperative relaying over a wireless link," IEEE Trans. Wireless Commun., vol. 11, no. 6, pp. 2076-2083, June 2012.

[9] O. Amin and L. Lampe, "Opportunistic energy efficient cooperative communication," IEEE Wireless Commun. Lett., vol. 1, no. 5, pp. 412 415, Oct. 2012.

[10] G. Lim and L. Cimini, "Energy-efficient cooperative relaying in heterogeneous radio access networks," IEEE Wireless Commun. Lett., vol. 1 no. 5, pp. 476-479, Oct. 2012.

[11] J. Choi, X. Weixi, D. To, Y. Wu, and X. Shugong, "On the energy efficiency of a relaying protocol with HARQ-IR and distributed cooperative beamforming," IEEE Trans. Wireless Commun., vol. 12, no. 2, pp. 769-781, Feb. 2013.

[12] S. Rini, E. Kurniawan, L. Ghaghanidze, and A. Goldsmith, "Energy efficient cooperative strategies for relay-assisted downlink cellular systems," IEEE J. Sel. Areas Commun., vol. 32, no. 11, pp. 1-15, Nov. 2014.

[13] O. Waqar, M. Imran, M. Dianati, and R. Tafazolli, "Energy consumption analysis and optimization of BER-constrained amplify-and-forward relay networks," IEEE Trans. Veh. Technol., vol. 63, no. 3, pp. 1256-1269, Mar. 2014.

[14] S. Huang, H. Chen, J. Cai, and F. Zhao, "Energy efficiency and spectralefficiency tradeoff in amplify-and-forward relay networks," IEEE Trans. Veh. Technol., vol. 62, no. 9, pp. 4366-4378, Nov. 2013.

[15] C. Sun, Y. Cen, and C. Yang, "Energy efficient OFDM relay systems," IEEE Trans. Commun., vol. 61, no. 5, pp. 1797-1809, May 2013.

[16] K. T. K. Cheung, S. Yang, and L. Hanzo, "Achieving maximum energy-efficiency in multi-relay OFDMA cellular networks: A fractional programming approach," IEEE Trans. Commun., vol. 61, no. 7, pp. 2746-2757, Jul. 2013.

[17] R. A. Loodaricheh, S. Mallick, and V. Bhargava, "Energy efficient resource allocation for OFDMA cellular networks with user cooperation and QoS provisioning," to appear in IEEE Trans. Wiresless Commun.

[18] A. Zappone, P. Cao, and E. Jorswieck, "Low-complexity energy efficiency optimization with statistical CSI in two-hop mimo systems," IEEE Signal Process. Lett., vol. 21, no. 11, Nov. 2014.

[19] — , "Energy efficiency optimization in relay-assisted MIMO systems with perfect and statistical CSI," IEEE Trans. Signal Process., vol. 2, no. 62, pp. 443-457, Jan. 2014.

[20] V.-A. Le, R.-A. Pitaval, S. D. Blostein, T. Riihonen, and R. Wichman, "One-bit CSI feedback selection schemes for energy-efficient multiuser and multirelay systems," IEEE Trans. Wireless Commun., vol. 12, no. 3, pp. 1149-1161, Mar. 2013.

[21] O. Amin, B. Gedik, and M. Uysal, "Channel estimation for amplifyand-forward relaying: Cascaded against disintegrated estimators," IET Commun., vol. 4, no. 10, pp. 1207-1216, Jul. 2010.

[22] V. Erceg, L. J. Greenstein, S. Y. Tjandra, S. R. Parkoff, A. Gupta, B. Kulic, A. A. Julius, and R. Bianchi, "An empirically based path loss model for wireless channels in suburban environments," IEEE $J$. Sel. Areas Commun., vol. 17, no. 7, pp. 1205-1211, Jul. 1999.

[23] W. Dinkelbach, "On nonlinear fractional programming," Management Science, vol. 13, no. 7, pp. 492-498, Mar. 1967.

[24] S. P. Boyd and L. Vandenberghe, Convex Optimization. Cambridge University Press, 2004. 